\title{
The Effects of Antibiotics, Metals, and Biotic Interactions on the Assembly of Taxonomically Diverse Single and Mixed Species Biofilms
}

\author{
Roshan Angoshtari1 ${ }^{1}$, Kim T. Scribner ${ }^{2}$, Terence L. Marsh ${ }^{1 *}$ \\ ${ }^{1}$ Department of Microbiology and Molecular Genetics, Michigan State University, East Lansing, MI, USA \\ ${ }^{2}$ Department of Fisheries and Wildlife, Michigan State University, East Lansing, MI, USA \\ Email: *marsht@msu.edu
}

How to cite this paper: Angoshtari, R., Scribner, K.T. and Marsh, T.L. (2020) The Effects of Antibiotics, Metals, and Biotic Interactions on the Assembly of Taxonomically Diverse Single and Mixed Species Biofilms. Advances in Microbiology, 10, 623-646. https://doi.org/10.4236/aim.2020.1012045

Received: November 2, 2020

Accepted: December 14, 2020

Published: December 17, 2020

Copyright () 2020 by author(s) and Scientific Research Publishing Inc. This work is licensed under the Creative Commons Attribution International License (CC BY 4.0).

http://creativecommons.org/licenses/by/4.0/

\begin{abstract}
To better understand the assembly of the sturgeon egg microbiome, we purified six bacterial isolates from eggs and characterized their ability to form biofilms under the stress of tobramycin, with and without exogenous protein. In experiments with single species biofilms, tobramycin reduced the metabolic activity of all isolates and increased biofilm biomass of three. The addition of exogenous protein to the assay countered the inhibition of biofilm and metabolic activity by tobramycin of Pseudomonas sp., Brevundimonas sp., Flavobacterium columnare and mixed biofilms of Pseudomonas-F. columnare and Brevundimonas-Hydrogenophaga. Two of the isolates (Pseudomonas spp.) that produced antimicrobial activity, were effective at reducing biofilm formation by Brevundimonas, but enhanced biofilm formation in other isolates. Increasing concentrations of $\mathrm{Mg}^{2+}$ had no effect on biofilm formation but $\mathrm{Ca}^{2+}$ enhanced biofilm formation of Pseudomonas aeruginosa PA01 (positive control) and Brevundimonas. Biofilm assembly by these two bacteria was inhibited by low concentrations of $\mathrm{Ni}^{2+}$. Mixed biofilms of Brevundimonas and Hydrogenophage consistently produced more robust biofilm than the strains in isolation, suggesting synergism. Established Brevundimonas biofilm appeared adept at recruiting pelagic Acidovorax and Hydrogenophaga into biofilm, suggesting that it plays an important role in the selection of species into the microbiome.
\end{abstract}

\section{Keywords}

Biofilm Assembly, Antimicrobial Resistance, Tobramycin, Metabolic Activity, Metal Resistance 


\section{Introduction}

The response of biofilm to stressors has been heavily investigated, primarily from the perspective of medicine and health. Biofilm is often viewed as an alternative life form of microbes that can provide some resistance to host defenses, antibiotics and other stressors [1]-[8]. In its most passive aspect, biofilm, by virtue of its macromolecular matrix, provides a barrier that protects the embedded cells from soluble stressors. For example, the cells of a $P$. aeruginosa biofilm are more resistant to tobramycin than planktonic cells [9] [10] and matrix encased bacteria cannot be opsonized as easily and are more resistant to polymorphonuclear cells [4] [11] [12]. There also appears to be "restricted penetration" of metals in biofilm matrices that accounts for an up to 600 times increase in resistance to toxic metals [13], although specific local conditions and time can play a large role [14] [15]. A more active role for biofilm has been demonstrated as well in the secretion of proteases, for example, that attack lactoferrin, a host-produced, bacterial growth-inhibiting protein [16]. More subtle is the strategy of Staphylococcus aureus biofilms that release lytic toxins that alter the differentiation of activated macrophages [17]. Biofilms have proven to be such a successful survival strategy that from $40 \%-80 \%$ of bacteria and archaea are thought to exist in quasi-structured matrix-bound communities [18].

Our work has focused on the biofilm of Lake Sturgeon (Ascipenser fluvescens) eggs as a model for biofilm assembly as well as for aspects of conservation. Regarding the former, eggs of aquatic vertebrates are expelled into the water in essentially a sterile state. Because these naive eggs are rapidly colonized by bacteria from the surrounding milieu, they provide an excellent view into the assembly of a natural, multispecies biofilm. With respect to conservation, mortality during early oncogenic stages can exceed 95\% for many fish species [19] [20] [21]. Lake Sturgeon is an endangered ancient species of fish with high egg mortality, in part the result of microbial activity [22]. Some endangered species, like the lake sturgeon, receive an assist from hatchery-reared juvenile supplements to the natural populations. More favorable outcomes in the hatchery will come from understanding the "rules of assembly" of the egg-associated microbes and the nature and composition of beneficial versus parasitic consortia that colonize these eggs.

Initial investigations into the sturgeon egg-associated microbiome included the isolation and characterization of over 100 bacterial isolates from the surface of sturgeon eggs as well as the phylogenetic characterization of the communities from healthy and moribund eggs [23]. Microbial community analysis allowed us to tentatively identify 3 - 4 phylotypes that were consistently present in healthy eggs and were also represented in our isolate collection [24]. These isolates included 1.) Acidovorax sp., a Betaproteobacteria in the Comamonadaceae family, 2.) Brevundimonas sp., an Alphaproteobacteria in the Caulobactereaceae family, 3.) Hydrogenophaga sp., a Betaproteobacteria, also in the Comamonadaceae family, and 4.) Massilia sp., a Betaproteobacteria in the Oxalobacteraceae family. Isolate characterization included screening for the production of antimicrobial 
activity in soft agar overlays [24] that led to the identification of two Pseudomonas spp. that produced strong antimicrobial activity against other members of the collection. More recent work has shown that three of these isolates, as well as the fish pathogen Flavobacterium columnare, formed substantially more robust biofilm in the presence of exogenous protein [25]. These isolates were utilized in this study to examine the effects of antimicrobials on biofilm assembly of single and double species biofilms, metabolic activity of tobramycin-stressed biofilms, and the resistance/sensitivity of biofilms to increasing concentrations of $\mathrm{Mg}^{2+}$, $\mathrm{Ca}^{2+}$ and $\mathrm{Ni}^{2+}$. Several of our isolates appear to interact synergistically when co-founding a biofilm and these mixed biofilms were examined as well. Finally, our studies identified Brevundimonas as a strong and collegial initiator of biofilm formation.

\section{Material and Methods}

\subsection{Media, Bacteria and Cultivation}

Lake Sturgeon egg isolates have been described previously [24] and included in this study were Massilia B13, Pseudomonas C22, Pseudomonas D2, Acidovorax F19, Hydrogenophaga F14, and Brevundimonas F16. These isolates were initially identified to genus level with partial 16S rRNA sequence [24]. GenBank accession numbers for the 16S rRNA sequence of the sturgeon isolates are: Massilia B13: KY075696, Pseudomonas C22: MH465524, Hydrogenophaga F14: MH465525, Brevundimonas F16: MH465526, and Acidovorax F19: MH465527. Pseudomonas C22, Acidovorax F19, Hydrogenophaga F14, and Brevundimonas F16 have since been refined with genomic data (Angoshtari \& Marsh, unpublished). The genome sequence of Pseudomonas C22 is closest to Pseudomonas fluorescence, strain 48D1 (Genbank accession \# MOBT01000000); Acidovorax F19 is closest to Acidovorax sp. CF316 (Genbank accession \# AKJX00000000); the genome sequence of Hydrogenophaga F14 is closest to Hydrogenophaga sp. RAC07 (Genbank accession \# CP016449). Finally, Brevundimonas F16 was originally identified as a Caulobacter based on partial sequence of 16S rRNA [24]. Genomic data now clearly identified this isolate as a Brevundimonas, closest to Brevundimonas subvibrioides strain 32-68-21 (Genbank accession \# NCEQ00000000). These genomic comparisons were made with Mash/MinHash as implemented in Patrick 3.6.7 [25] [26]. Because these "strains" have not been formally consecrated at the species level, we will refer to them as isolates throughout the manuscript. Pseudomonas aeruginosa PA01 was a gift of Dr. M. Bagdasarian (MSU) and Flavobacterium columnare 090702-1 was provided by Dr. Thomas Loch (MSU). Bacterial isolates were maintained at $-80^{\circ} \mathrm{C}$ in $20 \%$ glycerol solution and streaked onto R2A agar (Difco) several days prior to experiments. In experiments requiring broth, isolates were cultivated in R2broth, the composition of which is the same as R2A agar (Difco), minus the agar (Yeast extract $0.5 \mathrm{~g} / \mathrm{L}$, Proteose Peptone No. $30.5 \mathrm{~g} / \mathrm{L}$, Casamino Acids $0.5 \mathrm{~g} / \mathrm{L}$, Dextrose $0.5 \mathrm{~g} / \mathrm{L}$, Soluble Starch $0.5 \mathrm{~g} / \mathrm{L}$, Sodium Pyruvate $0.3 \mathrm{~g} / \mathrm{L}$, Dipotassium Phosphate 
$0.3 \mathrm{~g} / \mathrm{L}$, Magnesium Sulfate $0.05 \mathrm{~g} / \mathrm{L}$ ). To prepare broth with $2.5 \%$ milk protein, an autoclaved $5 \%$ solution of milk protein in water was added to an equal volume of $2 x$ R2Broth 30 minutes after autoclaving.

\subsection{Chemicals}

All solutions were prepared using water from a MilliQ water purification system fed with building Reverse Osmosis water. All chemicals were of molecular biology grade. $\mathrm{MgCl}_{2}, \mathrm{CaCl}_{2}$, and $\mathrm{NiCl}_{2}$ were purchased from Sigma-Aldrich. Milk Protein was purchased from Hardy Diagnostics and Crystal violet and Tobramycin, certified by the Biological Stain Commission, were purchased from Sigma-Aldrich. Koptec ${ }^{\mathrm{Tw}} 200$ Proof pure ethanol was purchased from Biochemistry Stores at Michigan State University. Glacial acetic acid ReagentPlus ${ }^{\circledast}$ was purchased from Sigma-Aldrich. A concentrated stock of tobramycin was filter sterilized ( $0.2 \mu \mathrm{m}$ Millipore) and then added to sterile R2Broth.

\subsection{Formation of Single- and Mixed-Species Biofilms}

In order to study single species biofilm formation, $50 \mu \mathrm{L}$ of an overnight bacterial culture grown in R2Broth was added to $100 \mu \mathrm{L}$ of sterile R2Broth in one well of a 96-well plate (Corning $\operatorname{costar}^{\circledR}$ 3595). Each condition was replicated 3 - 8 times depending on the experiment. Plates were sealed with sterile adhesive foil (VWR). We used $P$. aeruginosa PA01 as a positive control and 4 wells with un-inoculated sterile R2Broth as negative control. The average absorbance of the negative controls was subtracted from all samples. All plates were incubated at $25^{\circ} \mathrm{C}$ on a rotary shaker (100 RPM) for 48 or 96 hours, depending on the timeline of the experiment.

To investigate biofilm formation by double species mixed-cultures, $25 \mu \mathrm{L}$ of overnight culture of each isolate was added to the well along with $100 \mu \mathrm{l}$ of sterile R2Broth (3 - 4 replicates per condition based on the experiment). These plates were also incubated as above. In all experiments, pre- and post-incubation optical density at $600 \mathrm{~nm}$ was measured to confirm growth within the broth.

In addition to single- and mixed-species biofilm formation, we studied the effect of one isolate on the established biofilm of another isolate. In these experiments, $50 \mu \mathrm{L}$ of overnight culture of the first species was added to $100 \mu \mathrm{L}$ of sterile R2Broth in one well of a 96-well plate. After 24 - 48 hours of incubation the remaining pelagic cells and broth was removed, and the wells were washed $\mathrm{x} 3$ with sterile physiological saline. $50 \mu \mathrm{L}$ of the second species was then added along with $100 \mu \mathrm{L}$ of fresh sterile R2Broth and the plates were re-incubated for 24 - 48 hours, depending on the experiment.

\subsection{Tobramycin and Milk Protein Assays}

Tobramycin has been identified as an antibiofilm compound by numerous investigators [9] [10] [27] [28] [29] [30] [31]. Tobramycin was used at $5 \mu \mathrm{g} / \mathrm{mL}$. This concentration was derived from the literature as a level at which the cell 
numbers within a $P$. aeruginosa biofilm were reduced [31]. In addition, at this concentration the dominant mode of inhibition of Pseudomonas is at the level of the ribosome [32]. Because we were interested in the metabolic activity of biofilms, we tested the activity of the biofilm of all isolates and isolate combinations in the presence of $5 \mu \mathrm{g} / \mathrm{mL}$ tobramycin and found that activity was diminished in all cases. This indicated that tobramycin at $5 \mu \mathrm{g} / \mathrm{mL}$ was diffusing through the biofilm matrix and into cells. Regarding milk protein, previous work in our laboratory indicated that the addition of exogenous protein to cultures promoted substantial biofilm formation by some species [25]. Based on these studies, milk protein was tested at $2.5 \%$. To determine if tobramycin or milk protein influenced biofilm formation of our isolates, wells of 96-well microtiter plates containing $100 \mu \mathrm{l}$ R2Broth supplemented with either $2.5 \%$ milk protein or tobramycin $(5 \mu \mathrm{g} / \mathrm{mL})$ or both, were inoculated with single- or double-species mixtures and then incubated at $25^{\circ} \mathrm{C}$ on a rotary shaker (100 RPM) for 48 hours.

\subsection{Formation of Biofilm in the Presence of Metals}

In this set of experiments, biofilm formation by one or two isolates was tested in the presence of increasing concentrations of metals. R2Broth was supplemented with $\mathrm{CaCl}_{2}(10 \mu \mathrm{M}, 20 \mu \mathrm{M}, 50 \mu \mathrm{M}$, and $100 \mu \mathrm{M}), \mathrm{MgCl}_{2}(100 \mu \mathrm{M}, 200 \mu \mathrm{M}, 500$ $\mu \mathrm{M}$, and $1 \mathrm{mM})$, or $\mathrm{NiCl}_{2}(100 \mu \mathrm{M}, 200 \mu \mathrm{M}, 300 \mu \mathrm{M}$, and $400 \mu \mathrm{M})$. These metals and concentrations were selected because they have been reported to enhance biofilm formation [33] [34] [35] [36]. Biofilm biomass was measured using crystal violet assay after 48 hours of incubation at $25^{\circ} \mathrm{C}$ on a rotary shaker (100 RPM).

\subsection{Brevundimonas Titration Assays}

We measured biofilm formation of Hydrogenophaga F14 and Acidovorax F19 in co-culture with different starting concentrations of Brevundimonas F16 to determine the dependence of biofilm formation on Brevundimonas. Five $\mathrm{ml}$ of sterile distilled $\mathrm{H}_{2} \mathrm{O}$ was added to the surface of an R2A plate with a confluent culture of Brevundimonas. The cells were scrapped off the surface with a sterile bent glass rod and the cell suspension was made homogeneous by vortexing for 2 minutes. The cell suspension was centrifuged (10,000 RPM) for 30 minutes at $4^{\circ} \mathrm{C}$ and the resulting cell pellets were re-suspended in $5 \mathrm{~mL}$ fresh sterile $\mathrm{R} 2 \mathrm{Broth}$ and diluted in R2broth to the $\mathrm{OD} 600 \mathrm{~nm}$ of an overnight culture $(\sim 1.5)$. A dilution series was constructed using fresh R2Broth and resuspended cells at $0.3 \times, 0.2 \times, 0.1 \times, 0.05 \times$, and $0.01 \times$. Biofilms were established as described above for two isolates using overnight cultures of either Hydrogenophaga or Acidovorax with six different concentrations of Brevundimonas. Biofilm biomass was measured after 48 hours using crystal violet. Additionally, we tested the biofilm formation of mixed cultures using dead F16 cells to determine if an active culture of Brevundimonas was required for biofilm formation. This experiment was performed similar to the live titration assay except that after centrifuging Brevundimonas (10,000 RPM) for 30 minutes at $4^{\circ} \mathrm{C}$, the cell pellets were 
re-suspended in $5 \mathrm{~mL}$ of $80 \%$ ethanol and stored at $4^{\circ} \mathrm{C}$ for two weeks. After two weeks, $1 \mathrm{~mL}$ of the dead Brevundimonas F16/ethanol solution was centrifuged (10,000 RPM) for 10 minutes. The resulting dead cell pellet was then re-suspended in $1 \mathrm{~mL}$ fresh R2Broth. This step was repeated to completely remove residual $\mathrm{EtOH}$, after which the re-suspended ethanol killed cells were diluted $0.3 \times, 0.2 \times$, $0.1 \times, 0.05 \times$, and $0.01 \times$ in sterile R2Broth. The biofilm formation assay was then performed as described above using the original dilution (1x) as well as the dilution series of dead Brevundimonas F16 cells in combination with Hydrogenophaga F14 or Acidovorax F19. Biofilm biomass was measured after 48 hours using crystal violet assay.

\subsection{Crystal Violet Assay}

Biofilm biomass was measured using the crystal violet assay [37] [38]. Crystal violet is a triarylmethane dye that forms a bond with negatively charged molecules and polysaccharides on the surface of bacterial cells within the biofilm and/or the extracellular matrix [39]. All biofilm measurements were conducted in Corning Costar ${ }^{\mathrm{Tm}} 3595$ 96-well microtiter plates. Optical density measurements were performed at the beginning of each experiment after inoculation and at the end of incubation after transferring the supernatant to a new microtiter plate. Wells of incubated plates were gently washed $x 3$ with sterile $\mathrm{H}_{2} \mathrm{O}$ and the biofilm was stained with $200 \mu \mathrm{l}$ of a $0.2 \mu \mathrm{m}$ filtered, $0.1 \%$ crystal violet solution for 15 minutes while shaking at $100 \mathrm{RPM}$ on a rotary shaker at $25^{\circ} \mathrm{C}$. After staining, plates were rinsed twice in distilled water and inverted to dry completely. The biofilm bound dye was extracted with $200 \mu \mathrm{l}$ of $30 \%$ acetic acid accompanied by shaking at 100 RPM as above. Plates were read on a BioTek Epoch plate reader at $600 \mathrm{~nm}$. Wells were read x3, averaged, and the uninoculated control wells were subtracted from the absorbance of the sample wells.

\subsection{Resazurin Assay}

The resazurin assay [39] [40] [41] was used to measure metabolic activity within formed biofilms. Resazurin, also known as CellTiter-Blue (CTB) or Alamar Blue, is a blue non-fluorescent dye that reduces to the pink highly fluorescent resorufin by cellular metabolic activity. This effect increases proportionally with the number of cells that are metabolically active and, thus, serves as an indicator of metabolic activity of the population of cells within a biofilm. After incubation and the formation of biofilm, the wells were washed three times using sterile physiological saline. To each washed well, $100 \mu \mathrm{l}$ of physiological saline and $20 \mu \mathrm{l}$ of commercially available resazurin solution (CellTiter-Blue, CTB, Promega) were added [39]. The end point of the reaction was determined by measuring fluorescence ( $\lambda$ ex: $560 \mathrm{~nm}$ and $\lambda$ em: $590 \mathrm{~nm}$ ) after 60, 120, 180, 240, and 300 minutes of incubation $\left(25^{\circ} \mathrm{C}\right)$ [39]. Under our experimental conditions, the observed fluorescence strength approached its steady-state value by 180 minutes. Extended incubation time (240 and 300 minutes) did not result in any change of 
the fluorescence signal, therefore all incubations were for 180 minutes.

\subsection{Statistics}

The Student's t-test as implemented in Microsoft Excel (two sample assuming unequal variance) was used to evaluate dissimilarity between samples. In experiments where there was a mixed biofilm of two isolates, our null hypothesis was that the two populations of bacteria did not interact in any manner. In this way we compared two-isolate biofilms with the sum of the individual isolate biofilms. A "P" value of 0.05 or less indicated that the mixed biofilm differed from our null hypothesis. When challenging a 24-hour established biofilm with a second isolate, we used a biofilm with identical total incubation time derived from simultaneous inoculation of the two isolates as the control. For testing the effects of stressors (tobramycin or milk protein), the cognate sample without stressor(s) was used as the control.

\section{Results}

Over ten years ago we began an investigation into the microbial communities that attach to sturgeon eggs. As a model system, aquatic vertebrates offer a unique view into the assembly and biological relevance of microbial communities on the surface of eggs. When essentially sterile eggs are released from the female, they are engulfed by an environment with $10^{5}-10^{7}$ bacteria per $\mathrm{ml}$ and are rapidly colonized. Experimentally the investigator can control the environment surrounding the egg, measure physiological parameters and egg survival and correlate these with the egg-associated microbial community that assembled. Our previous work indicated that the egg-associated community was significantly different from the aquatic community, varied depending on the water temperature, increased in density as the egg matured and maintained unique populations of bacteria when healthy [23] [42]. Moreover, we purified over 100 bacterial isolates from the sturgeon egg and phylogenetically identified them, as well as characterizing their capacity to form biofilm and produce antimicrobial agents. Several of our isolates, Acidovorax F19, Brevundimonas F16, Hydrogenophaga F14 and Massilia B13, were correlated with higher survival rates of hatchery eggs and two Pseudomonas isolates, C22 \& D2, were identified as producers of antimicrobials as measured by soft agar overlay technique. How these isolates interact with each other and with the fish pathogen $F$. columnare in the formation of biofilm, and the resistance of this biofilm to antibiotics, are the subjects of these investigations.

In Figure 1, we present the effect of antimicrobial producing isolates Pseudomonas $\mathrm{C} 22$ and Pseudomonas D2 on biofilm formation. Under the standard conditions of our assay (growth in R2broth at $25^{\circ} \mathrm{C}$ ), biofilms produced by Pseudomonas C22 and D2, Hydrogenophaga and Massilia were lower than 0.1 A600 nm while Acidovorax and particularly Brevundimonas had relatively robust biofilms (Panel A). When isolates were challenged with Pseudomonas 


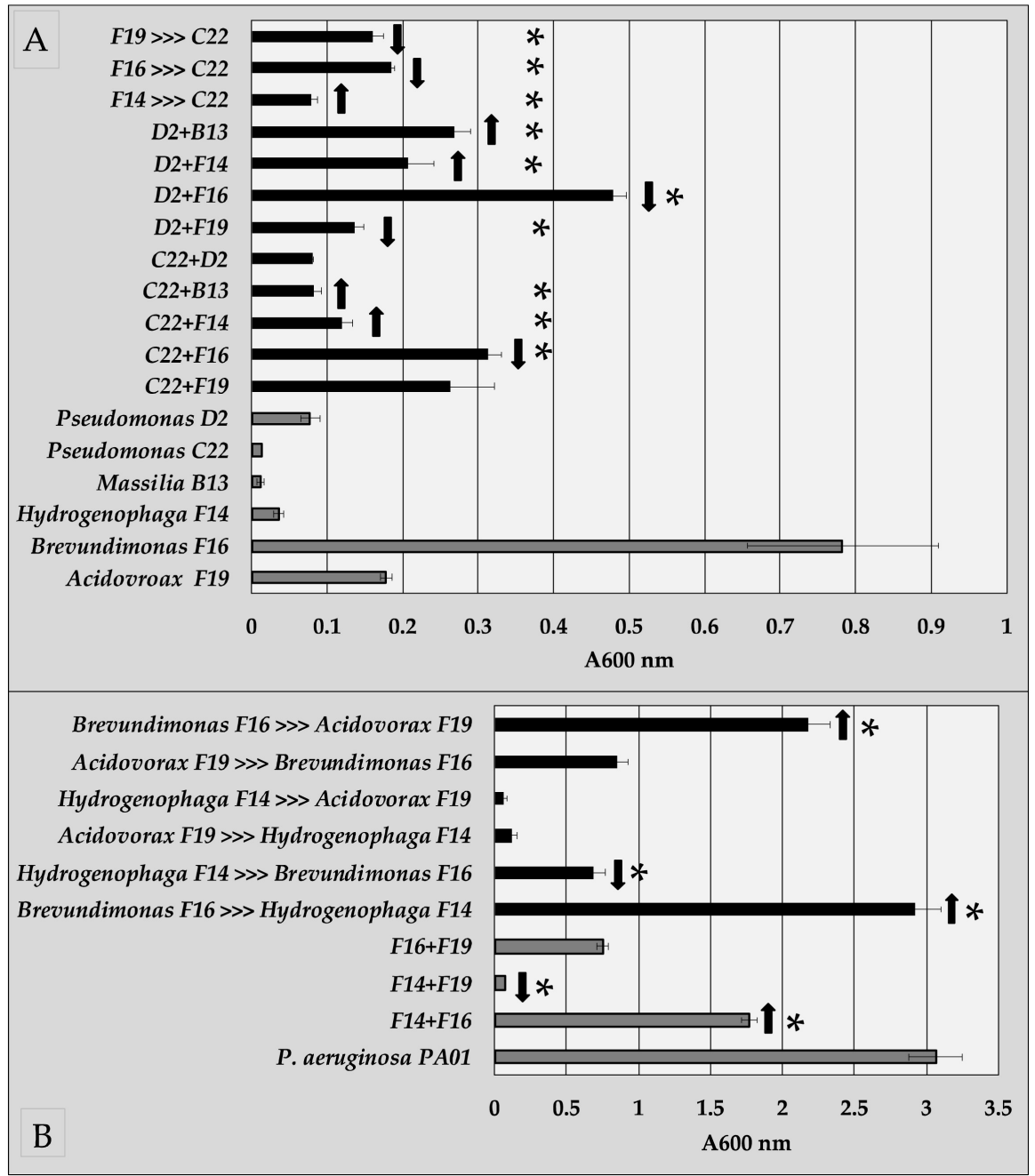

Figure 1. Crystal violet assays for single- and double-species biofilms. Panel (A). Single-species biofilms and biofilm assembly in the presence of antimicrobial-producing isolates C22 and D2. "Isolate X >> C22" indicates that the established biofilm of X was challenged after 24 hours by a fresh overnight culture of C22. Controls for the two-species biofilms (black bars) were the sums of cognate single-species biofilms (gray bars). Panel (B). Assembly of biofilm with two species (gray bars) and challenge of established single-species biofilms (black bars). Controls for the challenged biofilms (black bars) were the cognate two-species biofilms (gray bars). The sum of the single-species biofilms of Panel A served as the controls for two-species biofilms in Panel B (null hypothesis). The asterisks identify responses that were statistically different $(\mathrm{p}=0.05$ or less) from the controls. The arrows indicate an increase $(\uparrow)$ or decrease $(\downarrow)$ of absorbance compared to controls. Note the abscissa scale differences between the two panels.

$\mathrm{C} 22$, one of the antimicrobial-producing isolates, biofilm production varied greatly. Brevundimonas had substantially diminished biofilm while biofilms of Acidovorax, Hydrogenophaga and Massilia were modestly increased when incubated with Pseudomonas C22. When Pseudomonas D2 replaced C22, biofilm formation of both Acidivorax and Brevundimonas were reduced while that of Hydrogenophaga and Massilia were modestly increased. These changes were statistically relevant. As pointed out in the Material and Methods section, our 
null hypothesis was that the isolates did not interact in any manner. Therefore, the control for a two-species biofilm was the sum of the biofilms formed by single isolates. A Welch's t-test showing a high probability of difference between the two-species biofilm and the sum of the single isolate biofilms indicated departure from this null assumption. The combinations of Pseudomonas C22-Acidovorax F19 and Pseudomonas C22-Pseudomonas D2 produced biofilms at levels statistically indistinguishable from the null hypothesis. Pseudomonas C22 was of particular interest because it appeared to be the most aggressive isolate in soft agar overlays [42]. To determine if C22 could disrupt established biofilms of Acidovorax, Brevundimonas and Hydrogenophaga, C22 in fresh broth was added to established biofilms (24 hours) and incubated for an additional 24 hours (Top, Panel A). Acidovorax and Brevundomonas biofilms were reduced in this challenge but the biofilm of Hydrogenophaga was modestly increased. Massilia B13 is of interest to us as a potential probiotic but it forms clumps in liquid media and is therefore difficult to manipulate in these tests. For this reason, it was not carried into the remaining experiments. Panel B presents biofilm formation by two species where the focus is on temporal effects. Note here that the scale of the abscissa is different from Panel A, as more robust biofilms were detected. In this experiment, particular attention was paid to Brevundimonas, which appeared to enhance biofilm formation when combined with Hydrogenophaga, and under some conditions, Acidovorax. The combination of Brevundimonas and Hydrogenophaga routinely produced a more robust biofilm surpassing the simple sum of individual biofilms (null hypothesis). The combination of Acidovorax and Hydrogenophaga produced biofilm significantly less than single species tests, and the combination of Brevundimonas and Acidovorax produced biofilm amounts indistinguishable from the control. The sequence of addition of isolates in establishing mixed biofilms made a substantial difference in biofilm robustness. Acidovorax or Hydrogenophaga, when added to established biofilms of Brevundimonas, significantly increased biofilm biomass, nearly equivalent to the positive control, PA01. This suggests that Brevundimonas is able to recruit pelagic cells from the milieu. Other combinations where Brevundimonas was added as the second isolate to Acidovorax and the Acidovorax-Hydrogenophaga combination produced no substantial change in biofilm. A reduction in biofilm was detected when Brevundimonas was added to an established biofilm of Hydrogenophaga.

The effects of tobramycin and exogenous protein on the formation of biofilm are presented in Figure 2. Previous work by many investigators identified tobramycin as a disruptor [9] [27] [28] [29] [43] as well as an inducer of biofilm [44] [45]. As mentioned above, we have demonstrated that exogenous protein (milk protein in this example) provided a strong putative trigger for the formation of robust biofilm in select species, and we sought to extend this observation. In addition, the potential for altered resistance in mixed-species biofilms was examined, guided by previous work that indicated interactions between Pseudomonas $\mathrm{C} 22$ and $F$. columnare (a fish pathogen) and between Brevundimonas and Acidovorax or Hydrogenophaga. 


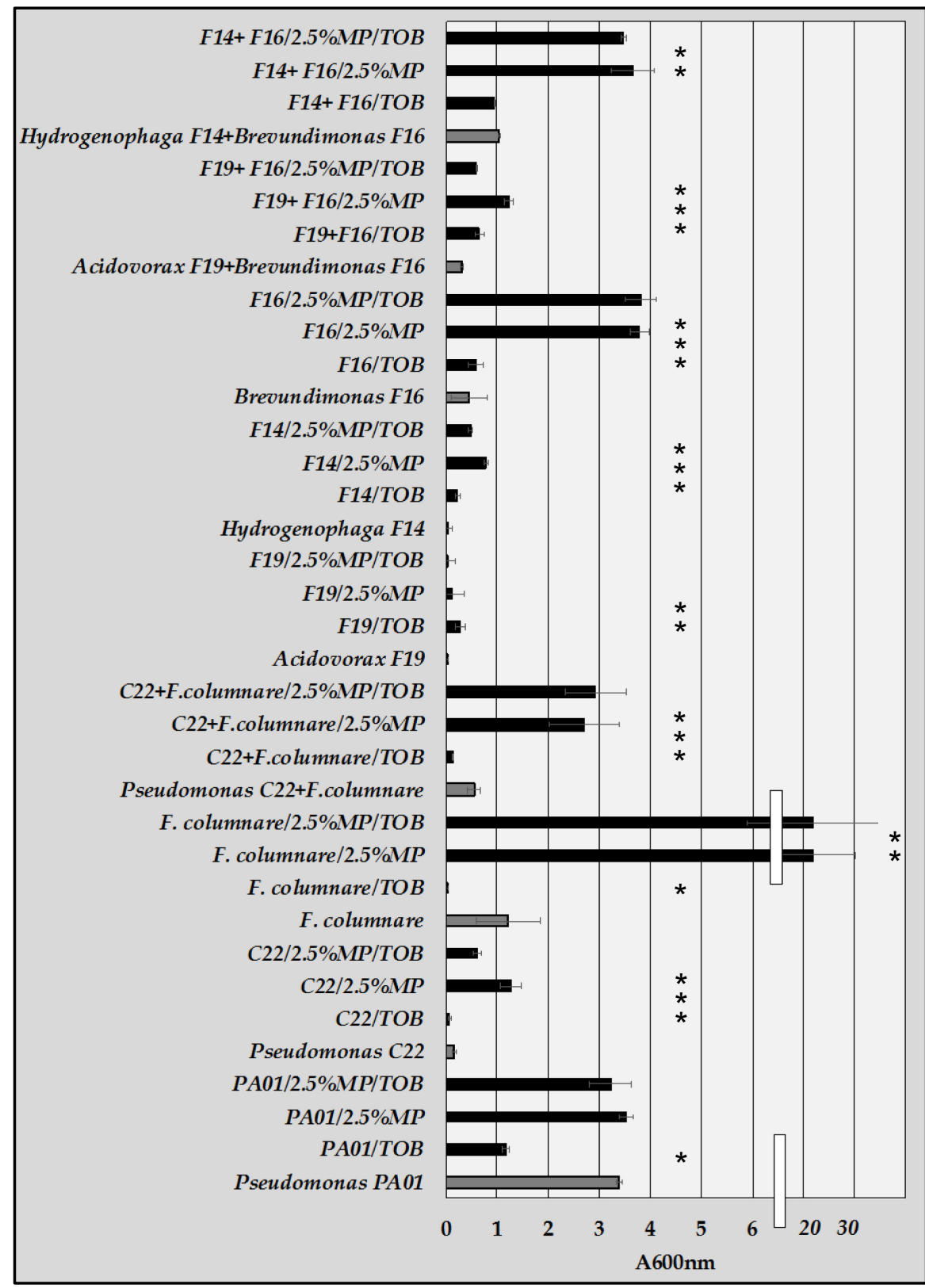

Figure 2. Crystal violet assays for single- and double-species biofilms. Tobramycin (TOB; $5 \mu \mathrm{g} / \mathrm{ml}$ ) and milk protein $(2.5 \%)$ were added at the start of the experiment. Note the discontinuity of the abscissa. The asterisks identify responses that were statistically different ( $p=0.05$ or less) from the unstressed controls (gray bars).

Regarding susceptibility of biofilm formation to tobramycin, only the isolate combination of Brevundimonas F16 and Hydrogenophaga F14 was unaffected. All other isolates and combinations were statistically different from the controls. The biofilms of Pseudomonas PA01, Pseudomonas C22, F. columnare and the combination C22-F. columnare were reduced while those of Acidovorax F19, Hydrogenophaga F14 and the combination of Acidovorax-Brevundimonas increased, compared to the unstressed controls. With the addition of milk protein to the incubations, only Pseudomonas PA01 was unaffected, as we have observed 
previously [25]. All isolates and two isolate combinations had statistically confirmed increases in biofilm, upon the addition of milk protein. In experiments where both milk protein and tobramycin were present, there were two responses. Some isolates saw complete amelioration of tobramycin inhibition when milk protein was present (Pseudomonas PA01, Brevundimonas, F. columnare, Pseudomonas C22-F. columnare and Brevundimonas-Hydrogenophaga) while the remaining isolates and combinations saw a partial amelioration.

Figure 3 presents the companion experiment to Figure 2 where the metabolic activities of single- and double-species biofilms were determined. There were

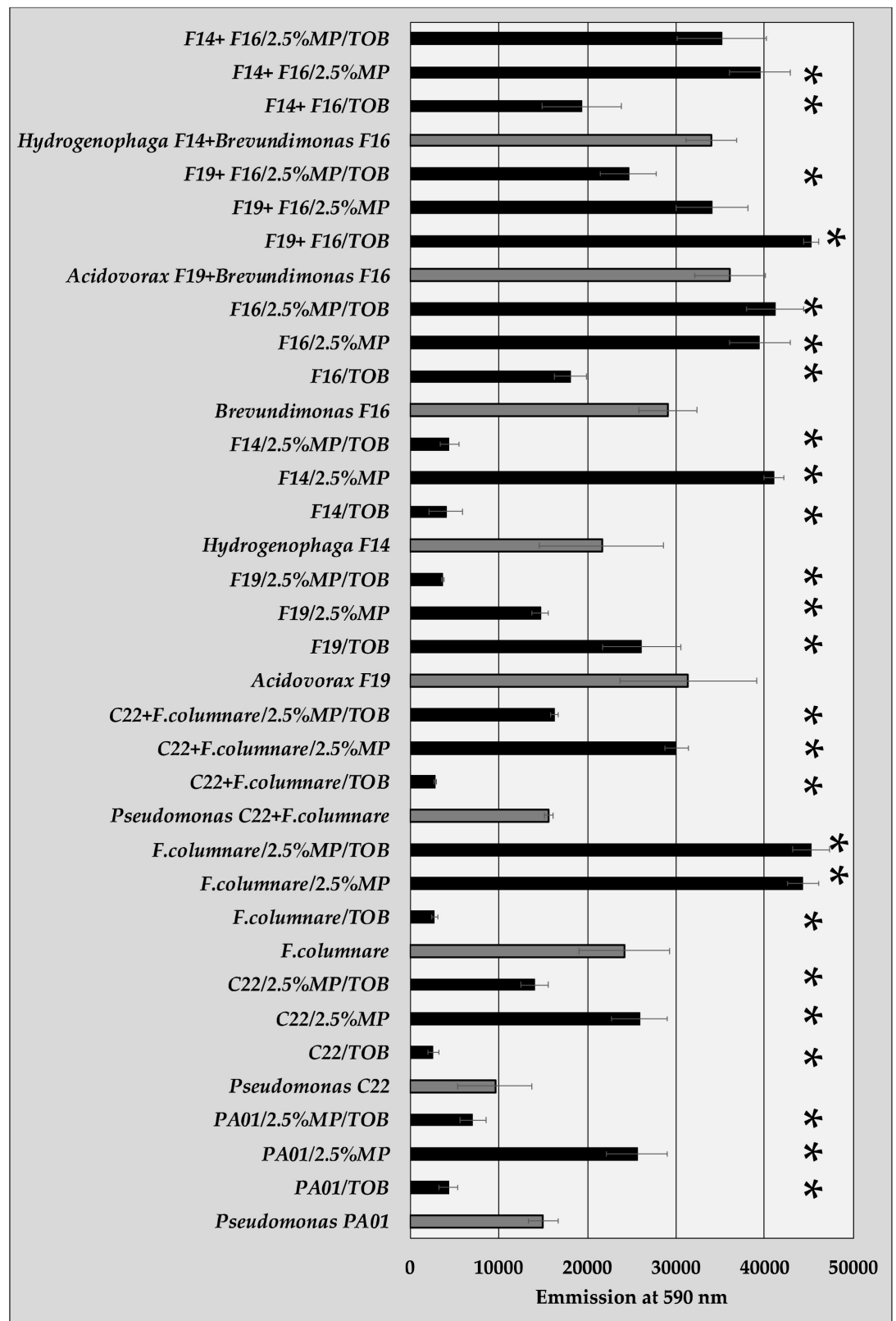

Figure 3. Resazurin assays of duplicate biofilms from experiments of Figure 2. The asterisks identify responses that were statistically different ( $p=0.05$ or less) from the unstressed controls (gray bars). 
only two cases where there was not statistical support for a departure from the control, unstressed state. All isolates and isolate combinations saw a statistically significant diminution of metabolic activity in the presence of tobramycin $(\mathrm{p}=$ 0.05 or less). All isolates and isolate combinations, with the exceptions of Acidovorax and the Acidovorax-Brevundimonas combination, saw an increase in metabolic activity upon addition of milk protein. With the addition of both milk protein and tobramycin, there were four phenotypic responses detected. F. columnare, Brevundimonas and the combination of Brevundimonas-Hydrogenophaga saw sharp, statistically significant reductions of metabolic activity with tobramycin, but complete amelioration of inhibition with the addition of milk protein. $P$. aeruginosa PA01 and $\mathrm{C} 22$ and the combination Pseudomonas C22-F. columnare were strongly inhibited by tobramycin, but partially recovered from inhibition with the addition of milk protein. Acidovorax was mildly (statistically significant) inhibited by tobramycin, more strongly inhibited by milk protein, and robustly inhibited by tobramycin + milk protein. Hydrogenophaga showed inhibition of activity with tobramycin, very strong activity when milk protein was present and then nearly complete inhibition of metabolic activity, when both tobramycin and milk protein were present.

The combination of measuring both biofilm biomass and metabolic activity provides unusual insights into the response of isolate and isolate combinations to changing environmental conditions. To better visualize the relationship between biofilm and metabolic activity, we computed the ratio of biofilm biomass (crystal violet staining) to metabolic activity (resazurin reduction) and plotted this as the fraction of the unamended cognate sample (Figure 4). Thus, the value for $P$. aeruginosa PA01 in the presence of tobramycin is 1.71 , indicating that the biomass: metabolic activity ratio is 1.71 times $P$. aeruginosa PA01 in the absence of the antibiotic. In the case of PA01, the metabolic activity has been disproportionally reduced compared to the biofilm. Only two samples had values less than 1.0, F. columnare + tobramycin and Pseudomonas PA01 + milk protein, indicating that the biomass: metabolic activity ratio was less than the isolate without supplements. Most of the remaining samples had values less than 10, indicating that the biofilm biomass had increased relative to the activity. Several samples were greater than 10 times their cognate control. Tobramycin alone produced nearly a 10 -fold increase in biofilm accompanied by a 10 -fold reduction in activity of Acidovorax and Hydrogenophaga, while milk protein produced substantial increases in biofilm biomass that were not matched by increases in activity in Acidovorax, Hydrogenophagaand F. columnare. Finally, the combination of milk protein and tobramycin yielded substantial increases in F. columnare, Acidovorax F19 and Hydrogenphaga F14. Of particular note was the robust response of Hydrogenophaga F14 where the biofilm increased 20-fold, but the activity was diminished 5-fold.

The effect of magnesium, calcium, and nickel on the formation of biofilms is presented in Figure 5. These metals were selected based on previous studies that suggested either an enhancement, in the case of magnesium and calcium [35] 


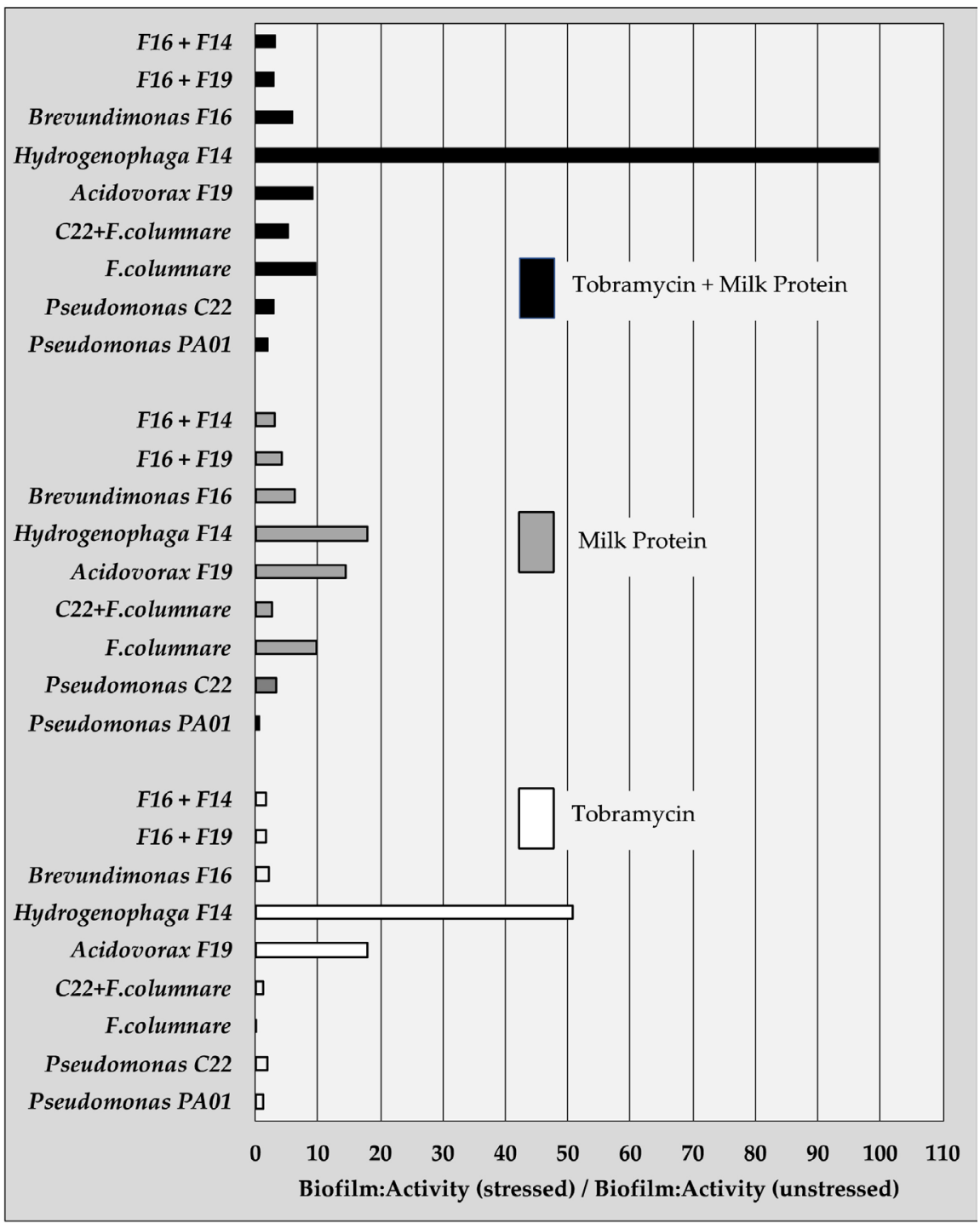

Figure 4. The ratio of biofilm biomass (CV) to metabolic activity (Resazurin) expressed as the fraction of stressed to the unstressed cognate sample.

[36], or stimulation, in the case of nickel [34], of biofilm formation. In the concentration range of $100 \mu \mathrm{M}-1 \mathrm{mM}, \mathrm{Mg}^{+}$had little effect on biofilm formation. Only Hydrogenophaga F14 and the combination of Brevundimonas-Pseudomonas C22 responded with a modest concentration-dependent increase in biofilm. The response to calcium $(1 \mu \mathrm{M}-100 \mu \mathrm{M})$ was more complex. Pseudomonas $\mathrm{C} 22$, Acidovorax F19, and the combination Pseudomonas C22 with Brevundimonas F16 showed no effects with calcium, but biofilm formation by $P$. aeruginosa PA01, Brevundimonas F16 and Hydrogenophaga F14 were modestly enhanced at the higher concentrations. The combinations of Brevundimonas with Hydrogenophaga or Acidovorax were moderately inhibited at higher calcium concentrations. Nickel was tested because of reports of biofilm stimulation in $E$. coli by subinhibitory concentrations [34]. Under the conditions of our assays in R2Broth, Nickel had little or no effect on biofilm formation by Pseudomonas C22, 


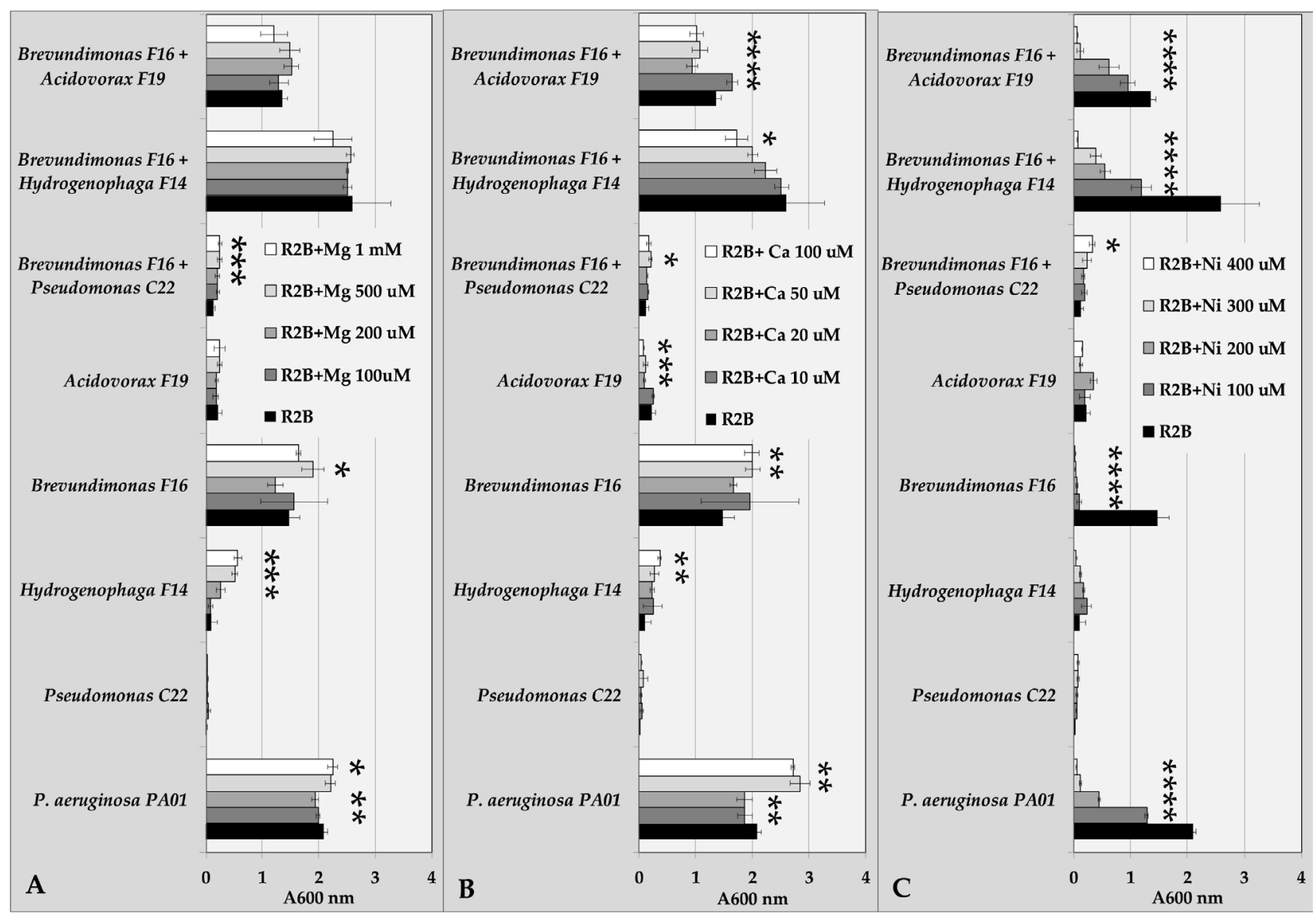

Figure 5. Crystal violet assay of biofilm formation in the presence of Metals. Panel (A). $\mathrm{MgCl}_{2}$ at $100 \mu \mathrm{M}, 200 \mu \mathrm{M}, 500 \mu \mathrm{M}$ and 1 mM. Panel (B). $\mathrm{CaCl}_{2}$ at $10 \mu \mathrm{M}, 20 \mu \mathrm{M}, 50 \mu \mathrm{M}$ and $100 \mu \mathrm{M}$. Panel (C). $\mathrm{NiCl}_{2}$ at $100 \mu \mathrm{M}, 200 \mu \mathrm{M}, 300 \mu \mathrm{M}$ and $400 \mu \mathrm{M}$. The asterisks identify responses that were statistically different ( $\mathrm{p}=0.05$ or less) from the controls (black bars).

Hydrogenophaga F14, Acidovorax F19 and the combination of Pseudomonas C22 and Brevundimonas F16. Nickel was, however, inhibitory to biofilm formation by $P$. aeruginosa PA01, Brevundimonas F16 and mixed biofilms with Brevundimonas and Hydrogenophaga or Acidovorax. Brevundimonas was particularly sensitive at even the lowest concentration $\left(100 \mu \mathrm{M} \mathrm{NiCl}_{2}\right)$.

In our investigations, Brevundimonas was an unusually robust biofilm former, particularly in the presence of exogenous protein. In addition, it facilitated biofilm formation in collaboration with other isolates, notably Hydrogenophaga and Acidovorax. To test its role in biofilm formation with these isolates, we titrated either Hydrogenophaga or Acidovorax with Brevundimonas in standard biofilm assays. The results are presented in Figure 6(A) and shows that the amount of biofilm of Hydrogenophaga or Acidovorax, when paired with Brevundimonas, was dependent on the starting concentration of Brevundimonas. Increasing concentrations of Brevundimonas increased the yield of biofilm. In Panel A the controls to establish statistical relevance were the two-species biofilms at full concentration. Ethanol-killed Brevundomonas was tested under the same conditions to determine if live cells were required (Figure 6(B)). No biofilm 


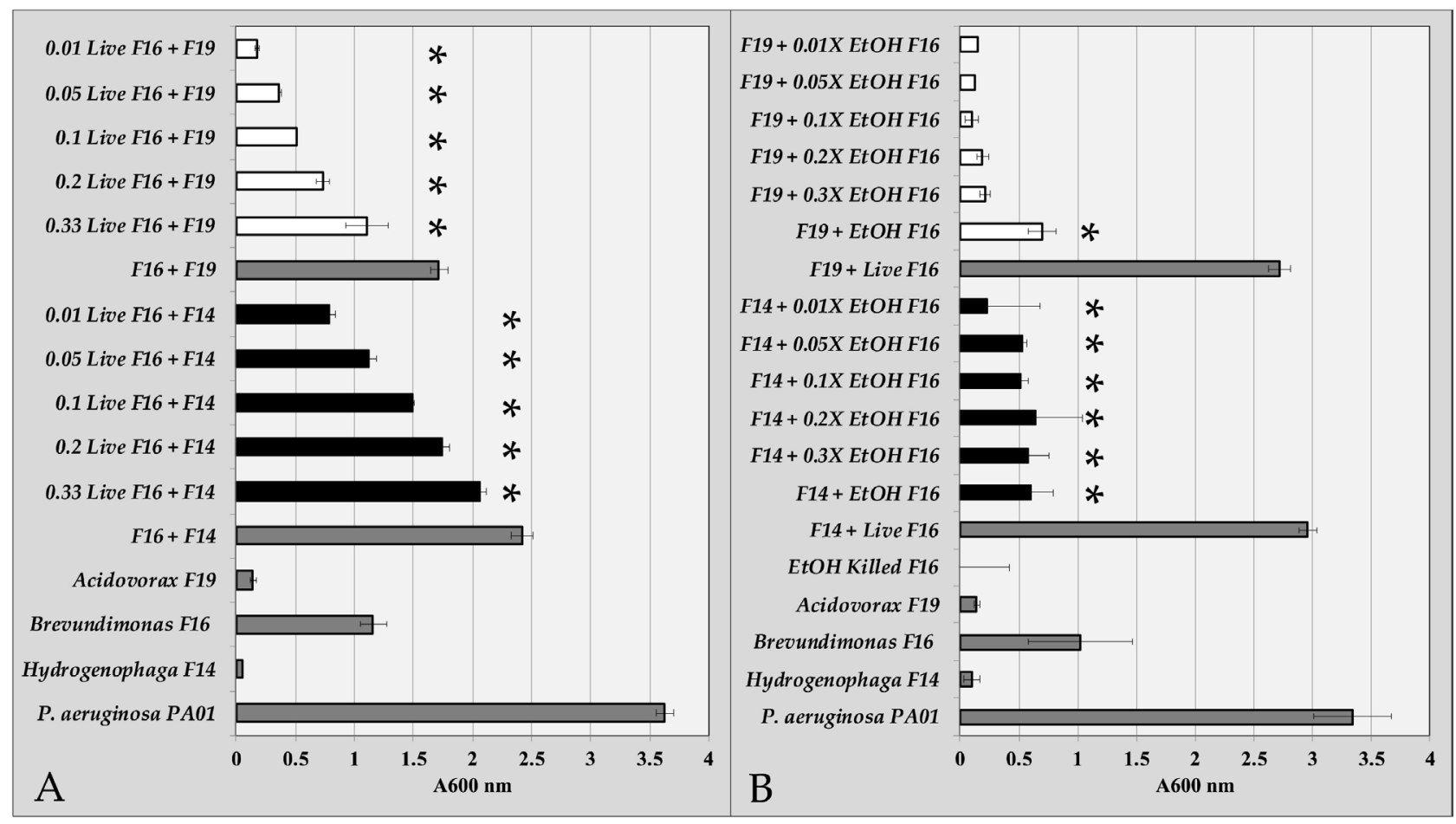

Figure 6. Two-species biofilm formation with Hydrogenophaga or Acidovorax in the presence of decreasing concentrations of Brevundimonas. P. aeruginosa PA01 is the positive control. Panel (A). Live Brevundimonas. The two-species biofilms at full concentration serve as controls for the dilution series. Panel (B). Ethanol-killed Brevundimonas. The asterisks identify responses that were statistically different ( $\mathrm{p}=0.05$ or less) from the controls.

was detected with Ethanol-killed Brevundimonas in the absence of a second strain. However, moderately enhance biofilm formation by Hydrogenophaga and Acidovorax was detected in the presence of Ethanol-killed Brevundimonas, although there was little concentration dependence. Note that in these experiments the controls to establish statistical relevance were the single-species biofilms of Hydrogenophaga or Acidovorax, inasmuch as Ethanol-killed Brevundimonas produced no measurable biofilm.

\section{Discussion}

The experiments reported herein describe the response of recently purified, undomesticated freshwater bacterial isolates to antibiotics and metals during the formation of biofilm. With the exception of P. aeruginosa PA01 and F. columnare, these isolates were purified from the microbiome associated with lake sturgeon eggs. In part, the variability in the amount of biofilm produced by these isolates under our experimental conditions reflects the phylogenetic and metabolic diversity of these bacteria. One isolate, Brevundimonas sp., produced a robust biofilm while the remaining five isolates made only modest amounts, compared to the $P$. aeruginosa PA01 positive control. In the formation of biofilm when two species are included in the inoculant, one of which produces an antimicrobial compound, the results were not straightforward. Our previous work indicated that both Pseudomonas C22 and D2 produced antimicrobial com- 
pounds in soft agar overlays that were inhibitory to Brevundimonas and Hydrogenophaga, but considerably less so to Acidovorax [24] and had no effect on Massilia. In addition, we also showed that established biofilm of Brevundimonas was significantly attacked by pelagic Pseudomonas C22 [24]. In this series of experiments when both an aggressive antibiotic producing isolate and a sensitive isolate were incubated together, biofilm formation did not necessarily correlate with the results from soft agar overlay. Brevundimonas had reduced biofilm when incubated with either Pseudomonas C22 or D2, consistent with results from a soft agar overlay challenge. In contrast, biofilm in mixed solutions of Hydrogenophaga and Massilia, with either Pseudomonas C22 or D2, was increased. The results with Acidovorax were mixed, showing an increase with Pseudomonas $\mathrm{C} 22$ but little change with D2. On testing the ability of Pseudomonas $\mathrm{C} 22$ to disrupt established biofilms, we found that it was effective against Brevundimonas, but not other isolates. Thus, even though Brevundimonas was accomplished in establishing a biofilm, that option was not effective against antimicrobials produced by the Pseudomonas isolates.

The results were equally complex when biofilm formation was challenged with tobramycin. In these experiments, biofilm assembly was conducted in the presence of tobramycin and the activity assays were conducted after the assembled biofilms had been washed. Tobramycin was not present during incubation with resazurin. Three isolates, $P$. aeruginosa PA01, F. columnare and Pseudomonas $\mathrm{C} 22$, saw a reduction in the amount of biofilm in the presence of tobramycin, whereas biofilms of Acidovorax, Brevundimonas and Hydrogenophaga increased. However, all six isolates appeared sensitive to tobramycin based on the reduction of metabolic activity. Tobramycin has been shown to inhibit bacterial growth via two mechanisms [32], inhibition of translation and disruption of the outer membrane. These experiments were conducted at a concentration of 5 $\mu \mathrm{g} / \mathrm{ml}$ (low-intermediate concentration), at which inhibition is thought to be via interference with translation. At higher concentrations $(>8 \mu \mathrm{g} / \mathrm{ml})$ disruption of the outer membrane is the primary mode of killing.

In mixed biofilms of Brevundimonas-Acidovorax, both biofilm and metabolic activity increased in the presence of tobramycin. Indeed, the biofilm biomass doubled indicating that this two-isolate alliance responded to the tobramycin challenge with increased metabolic activity not seen in the isolates individually. The nature of this synergism is not known. The opposite was seen with Pseudomonas $\mathrm{C} 22-F$. columnare where both biofilm and activity were reduced in the presence of tobramycin, consistent with the results of single species biofilms. The reduction of metabolic activity was substantially greater than the reduction of biofilm in the Brevundimonas-Hydrogenophaga mix but, in the absence of stressor, these two isolates together out-performed each isolate individually. We posit that the isolate combinations of Brevundimonas with either Aciovorax or Hydrogenophaga exhibit attributes that differed significantly from the isolates in isolation. Populations within a biofilm may be noncommunicative, antagonistic or cooperative where the nature of their interactions may stimulate functionality 
not present in single-species biofilms. This has been termed "emergent properties" of biofilms [46] and is thought to indicate synergisms between species [47]. The best example of this in our data was the interactions between Brevundimonas and Hydrogenophaga. When together, these isolates produced twice as much biofilm as the isolates individually. Mapping the intersections of each species' biofilm physiology will help to determine the essential metabolic features driving these synergisms.

As mentioned previously, milk protein was found to stimulate biofilm formation substantially in select species [25]. When $2.5 \%$ milk protein was applied as a stressor it elicited a more robust biofilm in Pseudomonas C22, F. columnare, Hydrogenophaga and Brevundimonas as well as an increase in metabolism. Milk protein also stimulated biofilm in Acidovorax but metabolic activity was reduced. Of interest was the response to tobramycin in the presence of milk protein which provided partial (Pseudomonas PA01, Pseudomonas C22, and C22-F. columnare) or complete (Brevundimonas and F. columnare) amelioration of the effects of tobramycin. This seemingly high protein concentration was not selected randomly. In previous work [25] we showed that select bacterial isolates produced abundant biofilm, as measured with the crystal violet assay, in the presence of relatively high concentrations of exogenous protein. We also demonstrated that the number of cells within the biofilm and the amount of protein incorporated into the matrix, increased, in response to exogenous protein.

Relatively high protein concentrations were selected in extended studies [25] to determine possible environmental stimuli of biofilm formation in Serratia marcescens. One potential habitat for this species is the human lung, where the concentration of only the surfactant proteins of alveolar fluid can be as high as $10 \%$ of the dry weight [48]. Similar high protein concentration exists in fish gills, targets of $F$. columnare. One interpretation of this is that in microenvironments with high protein concentration, the efficacy of tobramycin may be limited. Counter to this hypothesis is the observation that enhanced respiration, of the type we see upon addition of $2.5 \%$ milk protein, reduces the fraction of persister cells in biofilm of Mycobacterium tuberculosis, rendering it more susceptible to antibiotics [49]. Persister cells are present in biofilm and are thought to account, in part, for the resistance of biofilm to antibiotics [50] [51]. Vilchèze et al. [49] suggest that high metabolic rates and increasing consumption of $\mathrm{O}_{2}$ lead to fewer persister cells and greater production of reactive oxygen species. While reactive oxygen species have been invoked in the inactivation of antibiotics [52], clearly it does not explain the robust potency of tobramycin in Hydrogenophaga biofilm with milk protein where there was high metabolic activity. Thus, extending the results regarding persister cells from $M$. tuberculosis to these environmental isolates may be unwise. The metabolic diversity of the isolates in question may preclude a simple explanation.

It should be noted that in experiments where there were two isolates present in the establishment of a biofilm, we did not determine the phylotype composition, hence we do not know if one isolate substantially outcompeted another 
during the assembly process. Nonetheless, if we saw a substantial diminution of biofilm in mixed cultures compared with individual isolates, we can conclude that some form of antagonism was present. Similarly, a substantial boost in biofilm formation in mixed cultures suggests positive interactions between the two species. In a manuscript in preparation, the phylotypic composition of mixed biofilms was performed and we can report that in mixed biofilms of Brevundimonas with either Hydrogenophaga or Acidovorax, the phylotype distribution was approximately $55 \%$ Brevundimonas and $45 \%$ of the second isolate, under unstressed conditions similar to those used in the experiments described above. In a mixed biofilm of Pseudomonas C22 and F. columnare, the mixture was 90\% $\mathrm{C} 22$. Thus, even in a situation where there was potential for complete dominance by an antimicrobial producing isolate (C22), F. columnare maintained a foothold in the biofilm.

We selected magnesium, calcium and nickel to test because these metals enhance rather than inhibit biofilm formation. Both magnesium and calcium are divalent cations commonly found on the outer membrane of bacteria [53] [54] and appear to assist the structuring of biofilm at low concentrations [35] [36] [47]. Nickel has been shown to stimulate biofilm formation in E. coli at $100 \mu \mathrm{M}$ $\mathrm{Ni}^{2+}$ [34]. Metals are also toxic to biofilms [15] including nickel [55] [56]. In general, while we could detect statistically relevant differences in biofilm formation with magnesium and calcium, the effects were small. We found no substantial effect of magnesium on biofilm formation at relatively moderate concentrations. Calcium produced a modest stimulation of biofilm at the two highest concentrations for Pseudomonas PA01 and Brevundimonas and a moderate reduction of biofilm for two mixed species biofilms with Brevundimonas. Several hypotheses have been advanced to account for the influence of metals on biofilm including reducing surface charge and improve packing, bridging molecules and contributions to the structuring of the cell surface and conditioning the substratum [36]. Nickel proved to be the most interesting in that Brevundimonas and Pseudomonas PA01 appeared particularly sensitive. The concentrations we employed were based on $\mathrm{NiCl}_{2}$, hence the actual $\mathrm{Ni}^{2+}$ concentrations in the assays were $45 \mu \mathrm{M}, 90 \mu \mathrm{M}, 135 \mu \mathrm{M}$ and $180 \mu \mathrm{M}$. Thus, even at the lowest concentration, strong inhibition of biofilm formation was observed and Brevundimonas was particularly sensitive. While the sensitivity was attenuated in the mixed cultures with Acidovorax and Hydrogenophaga, at the higher concentrations of nickel the biofilm was reduced to barely detectable levels. Brevundimonas may be a critical target for nickel in the sense that our work indicated that Brevundimonas was instrumental in establishing mixed biofilms with emergent properties and higher concentrations of Brevundimonas translated into more robust biofilms. In stream systems, it has been observed that biofilm associated metals influence the microbial composition of the community [57]. All of our isolates were derived from freshwater systems, including Brevundimonas. Given the sensitivity of Brevundimonas to nickel, this group may be a sentinel for metal contamination. 
Brevundimonas, like the phylogenetically related genus Caulobacter, possesses a holdfast with a sticky terminus, located at one end of the rod-shaped cell [58] [59]. This structure is critical in establishing a connection to the substrata. Moreover, Brevundimonas biofilm actively recruited both Hydrogenophaga and Acidovorax from broth. We have also observed Brevundimonas induced cell-cell aggregation forming rosettes with Hydrogenophaga and Acidovorax under the light microscope (data not shown), similar to what is seen with Caulobacter [60]. Symbiotic relationships of Brevundimonas with other microbes have been identified [61] [62] and a recent report on nitrogen fixation in this genus [63] may explain in part, its collegiality. These attributes point to a key role for Brevundimonas in establishing biofilms in aquatic settings. Overall, our observations describe a deep complexity in the assembly, function and resistances of multispecies biofilms.

\section{Conclusions}

Our investigations into the structure and function of Sturgeon egg microbiomes were designed to assist in the development of hatchery protocols that reduce egg mortality caused by microbial activity. To that end, we have purified over 100 isolates from eggs and in preliminary studies, described antagonism between isolates and characterized biofilm formation [42]. The investigations presented here extend our description of interactions between isolates. By measuring the formation of biofilm comprised of one or two species in the presence and absence of stressors, we have identified several types of isolate interactions that may facilitate the formation of the egg's microbiome. We have identified significant sensitivity of some biofilm-forming isolates to two independently isolated Pseudomonas spp. that produce antimicrobial compounds in situ. In one case, the Pseudomonas isolate (C22) diminished the formation of a robust biofilm of F. columnare, a fish pathogen. While the metabolic activity of all tested isolates was diminished by tobramycin, three produced less biofilm and three produced more biofilm in response to the antibiotic. One of our isolates, Brevundimonas, formed abundant biofilm with increased metabolic activity in the presence of exogenous protein. In several cases, the presence of exogenous protein ameliorated the effects of tobramycin, suggesting that antimicrobial activity is conditional for these strains. Biofilm formation by both Brevundimonas and P. aeruginosa PA01 were exceptionally sensitive to low concentrations of nickel. Finally, we posit that Brevundimonas is a keystone species in the formation of multispecies biofilms based on the ability of established Brevundimonas biofilms to recruit pelagic Hydrogenophaga and Acidovorax and its collaborative interactions with Hydrogenophaga.

\section{Acknowledgements}

We thank the Department of Microbiology and the College of Natural Science for partial funding including a Thesis Completion grant. Other funding sources 
included the Michigan Dept Batyral Resources, Great Lakes Fishery Trust and the U.S. Fish and Wildlife Service Coastal Grants Program, Center for Water Sciences Water Cube initiative at MSU, MSU Ag Bio Research and the Department of Microbiology and Molecular Genetics at MSU. We thank Dr. M. Bagdasarian (MSU) and Dr. T. Loch at MSU for providing Pseudomonas aeruginosa PA01 and Flavobacterium columnare 090702-1, respectively.

\section{Author Contributions}

These experiments were conceptualized by RA, KTS and TLM. The methodology was worked out by RA and TLM and validation was performed by RA, KTS and TLM. Resources were provided by TLM and KTS. Data curation was by RA and TLM. RA was responsible for the first draft of the paper which constituted Chapter 2 of her Ph.D. thesis. TLM and KTS assisted in reviewing and editing of the first draft. Data visualization was by RA and TLM. TLM was the thesis advisor for RA and project administration and funding was through TLM and KTS. All authors have read and agreed to the published version of the manuscript.

\section{Ethical Approval}

This article contains no studies in which humans or metazoans were used as subjects.

\section{Conflicts of Interest}

The authors declare no conflict of interest. The funders had no role in the design of the study; in the collection, analyses, or interpretation of data; in the writing of the manuscript, or in the decision to publish the results.

\section{References}

[1] Roilides, E., et al. (2015) How Biofilms Evade Host Defenses. Microbiology Spectrum, 3, 1-10. https://doi.org/10.1128/9781555817466.ch14

[2] O’Toole, G., Kaplan, H. and Kolter, R. (2000) Biofilm Formation as Microbial Development. Annual Review of Microbiology, 54, 49-79. https://doi.org/10.1146/annurev.micro.54.1.49

[3] Mulcahy, L.R., Isabella, V.M. and Lewis, K. (2014) Pseudomonas aeruginosa Biofilms in Disease. Microbial Ecology, 68, 1-12. https://doi.org/10.1007/s00248-013-0297-x

[4] Kumar, A., et al. (2017) Biofilms: Survival and Defense Strategy for Pathogens. International Journal of Medical Microbiology, 307, 481-489. https://doi.org/10.1016/j.ijmm.2017.09.016

[5] Jamal, M., et al. (2018) Bacterial Biofilm and Associated Infections. Journal of the Chinese Medical Association, 81, 7-11. https://doi.org/10.1016/j.jcma.2017.07.012

[6] Jacques, M., Aragon, V. and Tremblay, Y.D. (2010) Biofilm Formation in Bacterial Pathogens of Veterinary Importance. Animal Health Research Reviews, 11, 97-121. https://doi.org/10.1017/S1466252310000149

[7] Hoiby, N., et al. (2011) The Clinical Impact of Bacterial Biofilms. International 
Journal of Oral Science, 3, 55-65. https://doi.org/10.4248/IJOS11026

[8] Fux, C.A., et al. (2005) Survival Strategies of Infectious Biofilms. Trends in Microbiology, 13, 34-40. https://doi.org/10.1016/j.tim.2004.11.010

[9] Anwar, H., et al. (1992) Dynamic Interactions of Biofilms of Mucoid Pseudomonas aeruginosa with Tobramycin and Piperacillin. Antimicrobial Agents and Chemotherapy, 36, 1208-1214. https://doi.org/10.1128/AAC.36.6.1208

[10] Anwar, H., Strap, J.L. and Costerton, J.W. (1992) Kinetic Interaction of Biofilm Cells of Staphylococcus aureus with Cephalexin and Tobramycin in a Chemostat System. Antimicrobial Agents and Chemotherapy, 36, 890-893. https://doi.org/10.1128/AAC.36.4.890

[11] Chorell, E., et al. (2012) Design and Synthesis of Fluorescent Pilicides and Curlicides: Bioactive Tools to Study Bacterial Virulence Mechanisms. Chemistry, 18, 4522-4532. https://doi.org/10.1002/chem.201103936

[12] Piatek, R., et al. (2013) Pilicides Inhibit the FGL Chaperone/Usher Assisted Biogenesis of the Dr Fimbrial Polyadhesin from Uropathogenic Escherichia coli. BMC Microbiology, 13, Article No. 131. https://doi.org/10.1186/1471-2180-13-131

[13] Teitzel, G.M. and Parsek, M.R. (2003) Heavy Metal Resistance of Biofilm and Planktonic Pseudomonas aeruginosa. Applied and Environmental Microbiology, 69, 2313-2320. https://doi.org/10.1128/AEM.69.4.2313-2320.2003

[14] Harrison, J.J., et al. (2004) Biofilm Susceptibility to Metal Toxicity. Environmental Microbiology, 6, 1220-1227. https://doi.org/10.1111/j.1462-2920.2004.00656.x

[15] Koechler, S., et al. (2015) Toxic Metal Resistance in Biofilms: Diversity of Microbial Responses and Their Evolution. Research in Microbiology, 166, 764-773. https://doi.org/10.1016/j.resmic.2015.03.008

[16] Schmidtchen, A., et al. (2002) Proteinases of Common Pathogenic Bacteria Degrade and Inactivate the Antibacterial Peptide LL-37. Molecular Microbiology, 46, 157-168. https://doi.org/10.1046/j.1365-2958.2002.03146.x

[17] Hanke, M.L. and Kielian, T. (2012) Deciphering Mechanisms of Staphylococcal Biofilm Evasion of Host Immunity. Frontiers in Cellular and Infection Microbiology, 2, 62. https://doi.org/10.3389/fcimb.2012.00062

[18] Bar-On, Y.M. and Milo, R. (2019) Towards a Quantitative View of the Global Ubiquity of Biofilms. Nature Reviews Microbiology, 17, 199-200.

https://doi.org/10.1038/s41579-019-0162-0

[19] Bouwes, N. and Luecke, C. (1997) The Fate of Bonneville Cisco Eggs in Bear Lake: Evaluating Mechanisms of Egg Loss. Transactions of the American Fisheries Society, 126, 240-247. https://doi.org/10.1577/1548-8659(1997)126<0240:TFOBCE >2.3.CO;2

[20] Fitzsimons, J.D., et al. (2007) Influence of Egg Predation and Physical Disturbance on Lake Trout Salvelinus namaycush Egg Mortality and Implications for Life-History Theory. Journal of Fish Biology, 71, 1-16. https://doi.org/10.1111/j.1095-8649.2007.01437.x

[21] Smith, S.J. and Marsden, J.E. (2009) Factors Affecting Sea Lamprey Egg Survival. North American Journal of Fisheries Management, 29, 859-868. https://doi.org/10.1577/M07-196.1

[22] Forsythe, P.S., et al. (2013) Experimental Assessment of the Magnitude and Sources of Lake Sturgeon Egg Mortality. Transactions of the American Fisheries Society, 142, 1005-1011. https://doi.org/10.1080/00028487.2013.790847

[23] Fujimoto, M. (2012) Microbial Succession on the Lake Sturgeon Egg Surface: Me- 
chanisms Shaping the Microbial Community Assembly during Succession and the Effect of Microbial Successional Processes on Host Life History Traits. Microbiology and Molecular Genetics, Michigan State University, East Lansing.

[24] Fujimoto, M., et al. (2018) Antagonistic Interactions and Biofilm Forming Capabilities among Bacterial Strains Isolated from the Egg Surfaces of Lake Sturgeon (Acipenser fulvescens). Microbial Ecology, 75, 22-37.

https://doi.org/10.1007/s00248-017-1013-z

[25] Ye, D., et al. (2020) Exogenous Protein as an Environmental Stimulus of Biofilm Formation in Select Bacterial Strains. Advances in Microbiology, 10, 123-144. https://doi.org/10.4236/aim.2020.103011

[26] Davis, J.J., et al. (2020) The PATRIC Bioinformatics Resource Center: Expanding Data and Analysis Capabilities. Nucleic Acids Research, 48, D606-D612.

[27] Yu, Q., et al. (2012) In Vitro Evaluation of Tobramycin and Aztreonam versus Pseudomonas aeruginosa Biofilms on Cystic Fibrosis-Derived Human Airway Epithelial Cells. Journal of Antimicrobial Chemotherapy, 67, 2673-2681. https://doi.org/10.1093/jac/dks296

[28] Tre-Hardy, M., et al. (2010) Efficacy of the Combination of Tobramycin and a Macrolide in an in Vitro Pseudomonas aeruginosa Mature Biofilm Model. Antimicrobial Agents and Chemotherapy, 54, 4409-4415.

https://doi.org/10.1128/AAC.00372-10

[29] Moreau-Marquis, S., O’Toole, G.A. and Stanton, B.A. (2009) Tobramycin and FDA-Approved Iron Chelators Eliminate Pseudomonas aeruginosa Biofilms on Cystic Fibrosis Cells. American Journal of Respiratory Cell and Molecular Biology, 41, 305-313. https://doi.org/10.1165/rcmb.2008-0299OC

[30] Kim, J., et al. (2008) Comparison of the Antimicrobial Effects of Chlorine, Silver Ion, and Tobramycin on Biofilm. Antimicrobial Agents and Chemotherapy, 52, 1446-1453. https://doi.org/10.1128/AAC.00054-07

[31] Anderson, G.G., et al. (2008) In Vitro Analysis of Tobramycin-Treated Pseudomonas aeruginosa Biofilms on Cystic Fibrosis-Derived Airway Epithelial Cells. Infection and Immunity, 76, 1423-1433. https://doi.org/10.1128/IAI.01373-07

[32] Bulitta, J.B., et al. (2015) Two Mechanisms of Killing of Pseudomonas aeruginosa by Tobramycin Assessed at Multiple Inocula via Mechanism-Based Modeling. Antimicrobial Agents and Chemotherapy, 59, 2315-2327.

https://doi.org/10.1128/AAC.04099-14

[33] Mulcahy, H. and Lewenza, S. (2011) Magnesium Limitation Is an Environmental Trigger of the Pseudomonas aeruginosa Biofilm Lifestyle. PLoS ONE, 6, e23307. https://doi.org/10.1371/journal.pone.0023307

[34] Perrin, C., et al. (2009) Nickel Promotes Biofilm Formation by Escherichia coli K-12 Strains That Produce Curli. Applied and Environmental Microbiology, 75, 1723-1733. https://doi.org/10.1128/AEM.02171-08

[35] Song, B. and Leff, L.G. (2006) Influence of Magnesium Ions on Biofilm Formation by Pseudomonas fluorescens. Microbiological Research, 161, 355-361. https://doi.org/10.1016/j.micres.2006.01.004

[36] Wang, T., Flint, S. and Palmer, J. (2019) Magnesium and Calcium Ions: Roles in Bacterial Cell Attachment and Biofilm Structure Maturation. Biofouling, 35, 959-974. https://doi.org/10.1080/08927014.2019.1674811

[37] Merritt, J.H., Kadouri, D.E. and O’Toole, G.A. (2011) Growing and Analyzing Static Biofilms. Current Protocols in Microbiology, 22, B.1.1-1B.1.18.

https://doi.org/10.1002/9780471729259.mc01b01s22 
[38] O’Toole, G.A. (2011) Microtiter Dish Biofilm Formation Assay. Journal of Visualized Experiments, 47, 2437. https://doi.org/10.3791/2437

[39] Peeters, E., Nelis, H.J. and Coenye, T. (2008) Comparison of Multiple Methods for Quantification of Microbial Biofilms Grown in Microtiter Plates. Journal of Microbiological Methods, 72, 157-165. https://doi.org/10.1016/j.mimet.2007.11.010

[40] Sandberg, M.E., et al. (2009) Pros and Cons of Using Resazurin Staining for Quantification of Viable Staphylococcus aureus Biofilms in a Screening Assay. Journal of Microbiological Methods, 78, 104-106. https://doi.org/10.1016/j.mimet.2009.04.014

[41] Van den Driessche, F., et al. (2014) Optimization of Resazurin-Based Viability Staining for Quantification of Microbial Biofilms. Journal of Microbiological Methods, 98, 31-34. https://doi.org/10.1016/j.mimet.2013.12.011

[42] Fujimoto, M., et al. (2013) Microbial Community Assembly and Succession on Lake Sturgeon Egg Surfaces as a Function of Simulated Spawning Stream Flow Rate. Microbial Ecology, 66, 500-511. https://doi.org/10.1007/s00248-013-0256-6

[43] Garo, E., et al. (2007) Asiatic Acid and Corosolic Acid Enhance the Susceptibility of Pseudomonas aeruginosa Biofilms to Tobramycin. Antimicrobial Agents and Chemotherapy, 51, 1813-1817. https://doi.org/10.1128/AAC.01037-06

[44] Elliott, D., Burns, J.L. and Hoffman, L.R. (2010) Exploratory Study of the Prevalence and Clinical Significance of Tobramycin-Mediated Biofilm Induction in Pseudomonas aeruginosa Isolates from Cystic Fibrosis Patients. Antimicrobial Agents and Chemotherapy, 54, 3024-3026. https://doi.org/10.1128/AAC.00102-10

[45] Hoffman, L.R., et al. (2005) Aminoglycoside Antibiotics Induce Bacterial Biofilm Formation. Nature, 436, 1171-1175. https://doi.org/10.1038/nature03912

[46] Flemming, H.-C., et al. (2016) Biofilms: An Emergent Form of Bacterial Life. Nature Reviews Microbiology, 14, 563-575. https://doi.org/10.1038/nrmicro.2016.94

[47] Sutherland, I.W. (1999) Biofilm Exopolysaccharides. In: Microbial Extracellular Polymeric Substances, Springer-Verlag, Berlin, 73-92. https://doi.org/10.1007/978-3-642-60147-7_4

[48] Goerke, J. (1998) Pulmonary Surfactant: Functions and Molecular Composition. Biochimica et Biophysica Acta Molecular Basis of Disease, 1408, 79-89. https://doi.org/10.1016/S0925-4439(98)00060-X

[49] Vilcheze, C., et al. (2017) Enhanced Respiration Prevents Drug Tolerance and Drug Resistance in Mycobacterium tuberculosis. Proceedings of the National Academy of Sciences of the United States of America, 114, 4495-4500. https://doi.org/10.1073/pnas.1704376114

[50] Lewis, K. (2005) Persister Cells and the Riddle of Biofilm Survival. Biochemistry (Moscow), 70, 267-274. https://doi.org/10.1007/s10541-005-0111-6

[51] Lewis, K. (2008) Multidrug Tolerance of Biofilms and Persister Cells. In: Romeo, T., Ed., Current Topics in Microbiology and Immunology, Springer-Verlag, Berlin, 107-132. https://doi.org/10.1007/978-3-540-75418-3_6

[52] Gujarathi, N.P. and Linden, J.C. (2005) Oxytetracycline Inactivation by Putative Reactive Oxygen Species Released to Nutrient Medium of Helianthus annuus Hairy Root Cultures. Biotechnology \& Bioengineering, 92, 393-402. https://doi.org/10.1002/bit.20698

[53] Ferris, F.G. and Beveridge, T.J. (1986) Physicochemical Roles of Soluble Metal Cations in the Outer Membrane of Escherichia coli K-1. Canadian Journal of Microbiology, 32, 594-601. https://doi.org/10.1139/m86-110

[54] Hoyle, B. and Beveridge, T.J. (1983) Binding of Metallic Ions to the Outer Mem- 
brane of Escherichia coli. Applied and Environmental Microbiology, 46, 749-752. https://doi.org/10.1128/AEM.46.3.749-752.1983

[55] Babich, H. and Stotzky, G. (1983) Toxicity of Nickel to Microbes: Environmental Aspects. Advances in Applied Microbiology, 29, 195-265. https://doi.org/10.1016/S0065-2164(08)70358-7

[56] Macomber, L. and Hausinger, R.P. (2011) Mechanisms of Nickel Toxicity in Microorganisms. Metallomics, 3, 1153-1162. https://doi.org/10.1039/c1mt00063b

[57] Ancion, P.Y., et al. (2013) Metal Concentrations in Stream Biofilm and Sediments and Their Potential to Explain Biofilm Microbial Community Structure. Environmental Pollution, 173, 117-124. https://doi.org/10.1016/j.envpol.2012.10.012

[58] Curtis, P.D. (2017) Stalk Formation of Brevundimonas and How It Compares to Caulobacter crescentus. PLoS ONE, 12, e0184063. https://doi.org/10.1371/journal.pone.0184063

[59] Sly, L.I., Cox, T.L. and Beckenham, T.B. (1999) The Phylogenetic Relationships of Caulobacter, Asticcacaulis and Brevundimonas Species and Their Taxonomic Implications. International Journal of Systematic Bacteriology, 49, 483-488.

https://doi.org/10.1099/00207713-49-2-483

[60] Poindexter, J.S. (2006) Dimorphic Prosthecate Bacteria: The Genera Caulobacter, Asticcacaulis, Hyphomicrobium, Pedomicrobium, Hyphomonas and Thiodendron. In: Dworkin, M., et al., Eds., Prokaryotes. A Handbook on the Biology of Bacteria, Vol. 5, Third Edition: Proteobacteria: Alpha and Beta Subclasses, Springer, New York, 72-90. https://doi.org/10.1007/0-387-30745-1_4

[61] Park, Y., et al. (2007) Growth Promotion of Chlorella ellipsoidea by Co-Inoculation with Brevundimonas sp. Isolated from the Microalga. Hydrobiologia, 598, 219-228. https://doi.org/10.1007/s10750-007-9152-8

[62] Pastore, M. and Sforza, E. (2018) Exploiting Symbiotic Interactions between Chlorella protothecoides and Brevundimonas diminuta for an Efficient Single-Step Urban Wastewater Treatment. Water Science and Technology, 78, 216-224. https://doi.org/10.2166/wst.2018.155

[63] Naqqash, T., et al. (2020) First Report of Diazotrophic Brevundimonas spp. as Growth Enhancer and Root Colonizer of Potato. Scientific Reports, 10, Article No. 12893. https://doi.org/10.1038/s41598-020-69782-6 